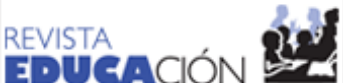

Revista Educación

ISSN: 0379-7082

ISSN: 2215-2644

revedu@gmail.com

Universidad de Costa Rica

Costa Rica

\section{Clase invertida para el desarrollo de la competencia: uso de la tecnología en estudiantes de preparatoria}

Flores, Linda Gladiola; Veytia Bucheli, María Guadalupe; Moreno Tapia, Javier

Clase invertida para el desarrollo de la competencia: uso de la tecnología en estudiantes de preparatoria

Revista Educación, vol. 44, núm. 1, 2020

Universidad de Costa Rica, Costa Rica

Disponible en: http://www.redalyc.org/articulo.oa?id=44060092022

DOI: https://doi.org/10.15517/revedu.v44i1.36961

Esta obra está bajo una Licencia Creative Commons Atribución-NoComercial-SinDerivar 3.0 Internacional. 


\title{
Clase invertida para el desarrollo de la competencia: uso de la tecnología en estudiantes de preparatoria
}

\author{
Flipped Classrooms for Skills Development: Use of Technology with High School Students
}

Linda Gladiola Flores

Universidad Autónoma del Estado de Hidalgo (UAEH), Redalyc: http://www.redalyc.org/articulo.oa?id=44060092022

DOI: https://doi.org/10.15517/revedu.v44i1.36961

México

lindaf2406@gmail.com

iD http://orcid.org/0000-0003-3463-1049

\author{
Maria Guadalupe Veytia Bucheli ${ }^{1}$ \\ Universidad Autónoma del Estado de Hidalgo (UAEH), \\ México \\ maria_veytia@uaeh.edu.mx \\ (iD http://orcid.org/0000-0002-1395-1644
}

\author{
Javier Moreno Tapia \\ Universidad Autónoma del Estado de Hidalgo (UAEH), \\ México \\ javier_moreno@uaeh.edu.mx \\ (D) http://orcid.org/0000-0003-4029-5440
}

Recepción: 03 Junio 2019

Aprobación: 05 Noviembre 2019

\section{RESUMEN:}

El empleo de las Tecnologías de la Información y la Comunicación (TIC) se ha incrementado en el ámbito educativo significativamente en la última década, una metodología que utiliza como recurso las TIC es la clase invertida, en la cual el estudiantado aplica la teoría y conceptos presentados en videos, y el profesorado desempeña un papel de guía y mediador. El objetivo del trabajo fue presentar los resultados de la aplicación de la clase invertida en la asignatura de Informática 1, en un grupo de estudiantado de la escuela preparatoria No. 1 de una institución pública del Estado de Hidalgo. Los sujetos de estudio fueron 90 estudiantes del primer semestre. Se empleó la metodología de investigación - acción en cuatro etapas: 1) Plan de acción, 2) Desarrollo de la acción, 3) Observación de la acción y 4) Reflexión. Se diseñaron, desarrollaron y evaluaron tres situaciones didácticas para trabajar la asignatura de informática que permitió fortalecer la competencia uso de la tecnología, a partir del empleo de la plataforma educativa Schoology, clips de Apple, MovieMaker y Youtube. Entre los principales hallazgos se encontró que el 92\% prefiere la clase invertida a clases tradicionales, el $8 \%$ prefiere el sistema tradicional. Mediante el empleo de esta metodología se logró atraer la atención de estudiantes con la grabación de videos por parte del cuerpo docente para la comprensión de los temas, además de que hubo un incremento de aprobación del estudiantado en esta asignatura.

Palabras clave: Clase invertida, Competencias, Preparatoria, Recursos Educativos, Tecnologías de la Información y la Comunicación, TIC.

\section{Abstract:}

Since the last decade, the use of Information and Communication Technologies (ICTs) in Education has been on the rise. Flipped classrooms use ICTs as resources in which students use videos to learn theories and concepts and the teacher plays the role of a facilitator or mediator. This study shows the results of how ICTs were used for a Computer Science 1 class by group of students

\section{NotAS DE AUTOR}


from high school No. 1 from the public school system in the Mexican state of Hidalgo. The subjects of the study included 90 students during the first semester. A four-stage research - action methodology was used that included: 1) an action plan, 2) development of the action, 3) observation of the action and 4) reflection. Three didactic situations involving Computer Science were designed, developed and assessed to strengthen the use of Technology using the Schoology educational platform, Apple Clips, Movie Maker and YouTube. Finding reveal that $92 \%$ of the students preferred flipped class compared to traditional classes. Only $8 \%$ preferred the traditional system. Technology helped maintain the attention of the students, particularly when the teacher used recorded video of the class to better understand the subject matter. As a result, student approval towards the subject also increased.

KeYwords: Flipped Classroom, Competence, High School, Educational Resources, Information and Communication Technologies, ITC.

\section{INTRODUCCIÓN}

Una educación de calidad requiere de un compromiso por parte de los diversos agentes que participan en el proceso de enseñanza aprendizaje, siendo los principales el profesorado y el estudiantado, en este caso, el profesorado debe adquirir responsabilidad y actitud de desaprender y reaprender, debido a los constantes cambios tecnológicos, educativos y requerimientos sociales, por lo tanto, es necesario contar con actitud innovadora, para lograr transformaciones que favorezcan y mejoren su actividad docente. En el caso del estudiantado, requiere de una actitud más participativa, activa y responsable en su proceso de aprendizaje, pues le implica el desarrollo de competencias sociales, tecnológicas y laborales las cuales le favorezcan en sus actividades futuras.

Con el avance de las tecnologías dentro del aula, y con antecedentes de éxito, es importante que el profesorado comience a innovar utilizando las Tecnologías de la Información y la Comunicación (TIC), logrando con esto que el estudiantado se sienta más motivado y con actitud positiva para ejecutar las tareas planteadas, y a su vez les generen el desarrollo de competencias que en Educación Media Superior, se requieren para cumplir con los aprendizajes esperados.

El objetivo del artículo es presentar los resultados de la aplicación de la clase invertida en la asignatura de Informática 1, en un grupo de estudiantado de la Escuela Preparatoria No. 1 de una institución pública del Estado de Hidalgo, y valorar después de su implementación las fortalezas y áreas de oportunidad en el desarrollo de la competencia uso de la tecnología.

\section{ESTADO DE LA CUESTIÓN}

Se desarrolló una investigación en libros y artículos, empleando el buscador Google Académico, y las bases de datos: Scielo, Redalyc y Latindex. Las palabras de búsqueda principales fueron: Flipped Classroom, clase invertida, y complementarias como: Nivel Básico, Bachillerato, Nivel Medio Superior, Nivel Superior. Se empleó una búsqueda avanzada considerando el periodo 2013 - 2018.

El estado de la cuestión muestra investigaciones que se han llevado a cabo en las instituciones educativas, empleando el modelo clase invertida, en niveles básico, medio superior y superior, con la finalidad de conocer el modo de planeación e intervención de este modelo, en los diferentes niveles. Esto a través de la revisión de artículos de revistas especializadas en educación, así como tesis a nivel de licenciatura y posgrado. La selección de las investigaciones se efectuó a partir de identificar diversos países de procedencia, aplicaciones en distintos niveles educativos, así como diversidad en las fuentes de procedencia, con el objetivo de tener variedad en los puntos de vista, así como en el proceso efectuado.

\section{- Concepto de clase invertida}

El modelo clase invertida constituye una innovación educativa la cual brinda buenos resultados. Entre los más mencionados se encuentra la motivación en el estudiantado, así como una mayor participación de estos en las actividades de aula, convirtiéndose en agentes activos y responsables 
de su aprendizaje; también logra fortalecer la comunicación entre docentes y estudiantes (Martín y Santiago, 2016; Valero, 2017; Melo y Sánchez, 2017; Sánchez, 2017; Ferriz, Sebastiá, y García, 2017).

Bergmann y Sams (2012), aseguran que invertir el aula significa que lo que tradicionalmente se hace en clase, ahora se ejecuta en casa y lo que tradicionalmente se efectúa como una tarea, es ahora completado en clase. En una clase tradicional, considerando la Taxonomía de Bloom (Churches, 2009), se promueven habilidades de nivel inferior como comprender y recordar; y en casa se promueven habilidades de nivel superior como aplicar, analizar, evaluar y crear, sin embargo, estas intentan ser desarrolladas por el estudiantado, de manera independiente. Lo que pretende la clase invertida es promover las habilidades de orden inferior a través de tareas y, las de orden superior en clase, con apoyo del profesorado y entre el estudiantado, permitiendo así, la apropiación de la habilidad de manera más significativa (Jordán, Pérez, y Sanabria, 2014; Valero, 2017).

- Países de procedencia de las investigaciones

Los países de procedencia de los trabajos revisados son: España (7 trabajos), Educador (dos trabajos) y México (dos trabajos). España, se considera como uno de los países que más ha implementado el modelo de clase invertida obteniendo buenos resultados en el rendimiento académico del estudiantado, (Martín y Santiago, 2016; Jordán et al., 2014; Valero, 2017; Sánchez, 2017; Ferriz et al., 2017; Massut, 2015). En los trabajos de Ecuador, el estudiantado se refiere al modelo como un proceso educativo que le genera mayor significado en su aprendizaje, a diferencia de la metodología tradicional (Barros y Martínez, 2018; Montenegro y González, 2017). Los trabajos de México, uno en el estado de Sonora en el que no se identificaron diferencias significativas en las evaluaciones pretest y postest de habilidades matemáticas (Madrid, Angulo, Prieto, Fernández y Olivares, 2018), y el otro de Hidalgo en donde refieren la importancia de un buen diseño instruccional y competencias docentes en TIC para la generación de motivación en el estudiantado y el correcto desarrollo de las actividades (González, Martínez, González y Bernal, 2015).

- El empleo de las TIC en el bachillerato

El estudio de Valero (2017) aplicado en una asignatura de TIC para primer semestre pretende demostrar la aplicación de una metodología de investigación acción con clase invertida para presentar una mejora educativa a partir de un área de oportunidad detectada previamente. Sánchez (2017) presenta un estudio del modelo clase invertida a través de una revisión teórica de su origen, evolución y aplicación en asignaturas de Economía, dentro del documento no presenta resultados de la aplicación.

Montenegro y González (2017), a partir del propio diseño de un sitio web con actividades interactivas buscan motivar al estudiantado en la asignatura de Historia en segundo año de bachillerato. Por otra parte, Massut (2015) diseña unidades de trabajo a partir de video tutoriales y actividades con clase invertida para presentar los temas de la asignatura de Matemáticas, analiza los trabajos desarrollados por el estudiantado así como el logro de sus competencias.

El estudio de Madrid et al. (2018) se lleva a cabo con el estudiantado de un curso propedéutico de habilidad matemática para el ingreso al bachillerato, con esta aplicación se pretende mejorar el rendimiento de los aspirantes en la habilidad matemática.

- TIC empleadas en la clase invertida

Las TIC no son un sustituto del profesorado, sino más bien una herramienta que, si es bien implementada, puede generar procesos de enseñanza aprendizaje muy enriquecedores. Al utilizarlas, el profesorado, puede tomar un rol de acompañante cognitivo y el estudiantado se convierte en el centro activo de su proceso de aprendizaje, convirtiéndose en entes que son capaces de seguir aprendiendo y de desenvolverse en distintos escenarios (Massut, 2015). En el modelo clase invertida es imprescindible utilizar las TIC por lo que a continuación se identifican las más mencionadas en los trabajos revisados. 
En el modelo de clase invertida, la TIC que se emplea con mayor frecuencia es el video tutorial, en el cual el profesorado brinda una explicación conceptual, y en algunos casos, explicación procedimental sobre el tema a tratar. En la mayoría de los estudios revisados, el profesorado elabora sus propios videos tutoriales, utilizando diversas plataformas para publicarlos. Algunos de ellos utilizan la plataforma de YouTube (Melo y Sánchez, 2017; Sánchez, 2017; González et al., 2015; Massut, 2015). Otros más, cuentan con sitios web propios o plataformas educativas utilizadas por sus instituciones educativas como Moodle o Edmodo, es en estos sitios en donde publican sus videos (Barros y Martínez, 2018; Montenegro y González, 2017; González et al., 2015).

Valero (2017) y Ferriz et al. (2017) indican que utilizan la plataforma Edpuzzle, la cual permite crear videocuestionarios para comprobar la comprensión del estudiantado durante el transcurso del video, otra característica importante es que evita que cada estudiante avance el video. En el caso de Martín y Santiago (2016), además de los videos elaborados por los propios docentes, también utilizan algunos ya publicados en YouTube.

Otra TIC identificada en algunos trabajos es Socrative, una herramienta en línea que permite crear cuestionarios con preguntas directas o crear concursos de preguntas (Martín y Santiago, 2016; Valero, 2017), el uso de esta herramienta requiere que el estudiantado cuente con algún dispositivo móvil dentro del aula para llevar a cabo la actividad.

Los servicios en línea que ofrece Google como presentaciones digitales, formularios para crear cuestionarios en línea y sitios web para publicar un diseño instruccional y materiales digitales, son utilizados también como herramientas de apoyo para la implementación del modelo clase invertida (Martín y Santiago, 2016; Jordán, et al., 2014; Valero, 2017; Massut, 2015).

- Ventajas de la clase invertida

En diversos estudios se ha observado el incremento de la motivación y la participación, así como mejora en el rendimiento académico por parte del estudiantado al ofrecerles nuevas formas de aprender (Fainholc, 2009; Madrid et al., 2018).

También se le responsabiliza al estudiantado por su aprendizaje, convirtiéndolo en un agente activo, que participa, aprende del profesorado, de sus compañeros y, además colabora en el aprendizaje de los demás (Martín y Santiago, 2016; Valero, 2017; Melo y Sánchez, 2017; Sánchez, 2017; Massut, 2015).

García (2013) comenta que genera espacios educativos de confianza, dinámicos, de reflexión y de trabajo colaborativo. Así mismo, brinda un aprendizaje al estudiantado a su propio ritmo.

El apoyo del profesorado es vital en este modelo, principalmente desempeña un rol de mediador y aclara dudas, lo cual favorece el desempeño del estudiantado y este adquiere mayor responsabilidad y logra sentirse en confianza para externar su punto de vista (Valero, 2017; Martín y Santiago, 2016; Melo y Sánchez, 2017).

En el caso de los trabajos comparados, se logró identificar una mejoría significativa en el aprovechamiento académico del estudiantado, con respecto a quienes conservaban una metodología tradicional (Barros y Martínez, 2018). No obstante, en otros casos comparados, las diferencias no resultaron significativas (Madrid et al., 2018). Esto puede darse por varias razones, toda innovación o estrategia educativa que se lleve a cabo, así cuente con los mejores antecedentes de éxito, siempre va a requerir de una actitud de participación, tiempo y disposición por parte, tanto del profesorado como del estudiantado (González et al., 2015).

- El empleo de los videos tutoriales en la clase invertida

En el caso de los videos tutoriales utilizados en la implementación de los trabajos revisados, se consideran de gran apoyo para estudiantes, que aunque les implica mayor dedicación y tiempo para prepararse antes de clase, se muestran comentarios positivos. Un aporte importante a considerar, es la aplicación de cuestionarios al estudiantado después de la revisión de los videos. Esto, le permite al 
profesorado identificar las dudas y fallas para así, preparar material que le permita atenderlas al llegar a la sesión presencial (Valero, 2017).

Dentro de las áreas de oportunidad identificadas, se encuentra la duración de los videos, que no sean muy extensos, pues provoca la pérdida de concentración y aburrimiento, por lo que se sugiere un tiempo no mayor a 10 minutos y mostrar una exposición clara (Jordán et al., 2014; Valero, 2017).

- Fortalezas y áreas de oportunidad de la clase invertida

Según García (2013), el aula invertida ha mostrado tener beneficios y efectividad, por lo que se está recibiendo una mayor atención en el ámbito educativo. Es una estrategia que fomenta la curiosidad y el trabajo colaborativo del alumnado.

El modelo clase invertida ofrece un aprendizaje constructivista, utilizan actividades interactivas en las cuales el estudiantado juega un papel activo (Massut, 2015).

Sin duda, el modelo clase invertida, ofrece muchos beneficios en su implementación, no obstante, también requiere de un gran compromiso. Por una parte, por la institución educativa al proporcionar la infraestructura necesaria, posteriormente un gran tiempo de planeación por parte del profesorado, para brindar estrategias innovadoras y materiales didácticos de calidad y finalmente, un compromiso por parte del estudiantado, al adquirir un nuevo papel, que le ofrezca mayor responsabilidad y protagonismo.

\section{REFERENTES CONCEPTUALES O MARCo TEÓRICO}

El desarrollo de este apartado se encuentra organizado en tres bloques, el primero aborda la importancia del triángulo didáctico que se genera entre el saber, el profesorado y el estudiantado, en el segundo se destaca la vinculación de las TIC con la educación, y finalmente se profundiza en la clase invertida, sus características, definiciones y los roles que desempeña el estudiantado.

- Triángulo didáctico

La enseñanza refiere a reconocer a un sujeto que enseña, algo que se enseña y a alguien a quien enseña. El propósito es, por lo tanto, ayudar a alguien a que aprenda algo, estos tres elementos resultan importantes en la enseñanza y son indispensables (Ibáñez, 2007).

El triángulo didáctico implica más que mencionar la interacción entre los tres agentes mencionados, sino que requiere de un mayor análisis. No solo es una adquisición de información, sino aprender a hacer o resolver y, de manera innovadora. También son fundamentales las habilidades y destrezas del profesorado, entre las más importantes, dominar su disciplina, conocimiento en teoría de la educación, didáctica de la disciplina que enseña, discurso didáctico y aterrizar los aprendizajes a situaciones reales, considerando también el uso de las tecnologías que, sin duda, le son de gran utilidad (Díaz, 2010; Ibáñez, 2007; Salas, 2016).

Ibáñez (2007) también menciona que el conocimiento es parte fundamental en el proceso educativo, sin embargo, no puede ser definido como una cosa, debido a que no posee propiedades que lo definan como una entidad concreta o sustancial. Resulta complejo definirse como un factor del que pueda depender alguna acción sobre otros agentes. El conocimiento de dominio y esto es diverso para cada ser humano, dado que participa una gran cantidad de factores como su contexto social, familiar, escolar, entre otros. Además de tener identificados a los agentes que intervienen, será importante identificar las relaciones entre ellos, así como la interacción que se desarrolla en su proceso de enseñanza aprendizaje y el grado de desempeño que estos agentes ejecuten.

- TIC en educación

Cabero y Ruiz (2017), define a las TIC como 
instrumentos técnicos que giran en torno a los nuevos descubrimientos de la información. Medios eléctricos que crean, almacenan, recuperan y transmiten información de forma rápida, en gran cantidad, y lo hacen combinando diferentes tipos de códigos en una realidad hipermedia (p. 26).

Además de ser los instrumentos que permiten la interacción entre el mundo digital y los individuos.

La inclusión de las TIC en entornos educativos, se ha convertido en una obligación, pero sobre todo una necesidad para generar procesos de innovación los cuales permitan elevar la calidad, equidad, acceso y pertinencia en la educación superior (Salas, 2016). Pero también se convierte en un factor importante que atender en los niveles de educación media superior, en donde el estudiantado debe desarrollar y adquirir las competencias necesarias para su correcto desempeño, en sus estudios universitarios y laborales.

- Atributos de las TIC

Las TIC cuentan con propiedades o atributos de gran valor para el profesorado que desean innovar en sus procesos de enseñanza aprendizaje, a continuación, se presentan los más relevantes (Gómez, 2016):

a) Formalismo. Implica previsión y planificación de las acciones. Favorece la toma de conciencia y la autorregulación.

b) Interactividad. Permite una relación más activa y contingente con la información. Potencia el protagonismo del aprendiz. Facilita la adaptación a distintos ritmos de aprendizaje. Tiene efectos positivos para la motivación y la autoestima.

c) Dinamismo. Ayuda a trabajar con simulaciones de situaciones reales. Permite interactuar con realidades virtuales. Favorece la exploración y la experimentación.

d) Multimedia. Permite la integración, la complementariedad y el tránsito entre diferentes sistemas y formatos de representación. Facilita la generalización del aprendizaje.

e) Hipermedia. Comporta la posibilidad de establecer formas diversas y flexibles de organización de las informaciones, estableciendo relaciones múltiples y diversas entre ellas. Facilita la autonomía, la exploración y la indagación. Potencia el protagonismo del aprendiz.

f) Conectividad. Permite el trabajo en red de agentes educativos y aprendices. Abre nuevas posibilidades al trabajo grupal y colaborativo. Facilita la diversificación, en cantidad y calidad, de las ayudas que los agentes educativos ofrecen a los aprendices.

Los atributos de las TIC se convierten en aliados de los agentes educativos, sobre todo del profesorado que desean innovar en su clase, con nuevas formas de enseñar. Actualmente existen nuevos modelos, que si bien, no reemplazan la metodología de enseñanza tradicional, sino que transforman los procesos, para brindar una mejor atención a las exigencias sociales y educativas (Vaughan, Cleveland y Garrison, 2013; Bergmann y Sams, 2012).

- Clase invertida

El modelo clase invertida surgió a partir de dos docentes estadounidenses que deseaban ayudar a sus estudiantes cuando faltaban a clases o, que presentaban complicaciones a la hora de comprender los temas teóricos. El profesorado comenzó a invertir sus actividades, el concepto de invertir la clase es lo que tradicionalmente se hace en clase ahora se hace en casa, y lo que tradicionalmente se hace de tarea se completa ahora en clase (Bergmann y Sams, 2012).

Este modelo se apoya mucho de las TIC para el logro de sus objetivos, pues a través de videos, el docente transmite, con un discurso didáctico, los conocimientos conceptuales, pero también pueden ser procedimentales para mostrar ejemplos del tema a abordar. En este momento, el estudiantado puede repetir o pausar el video para hacer notas y anotar dudas sobre el tema (Valero, 2017). 
Posterior a esto, y dentro del aula, se inicia con una discusión sobre el video, también pueden contestarse cuestionarios y atenderse dudas particulares sobre lo visto en el video. Finalmente, se asignan actividades para trabajar en el aula como pueden ser resolución de problemas o llevar a cabo proyectos (Ver Figura 1), en los que el estudiantado toma un papel activo que le permita trabajar y aprender de manera colaborativa (Bergmann y Sams, 2012).

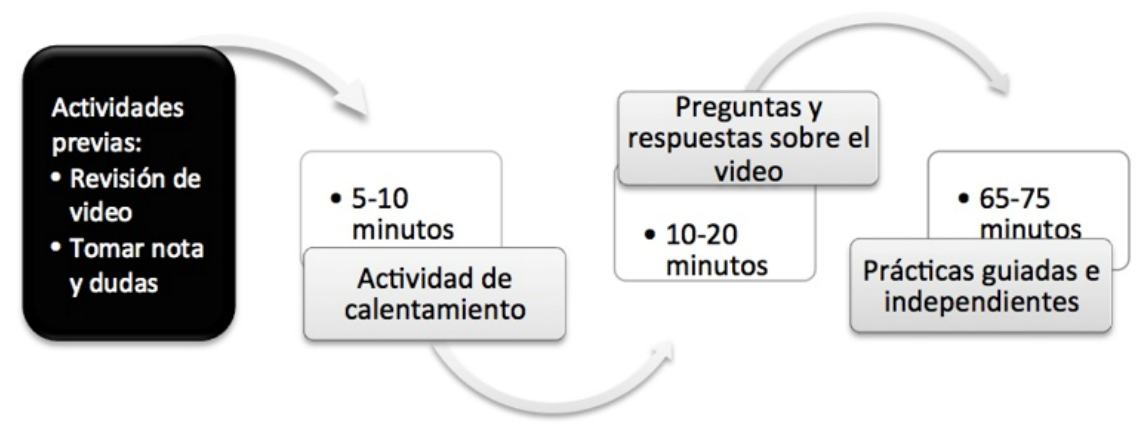

\author{
FIGURA 1 \\ Tiempo de clase invertida \\ Fuente: Elaboración propia a partir de (Bergmann y Sams, 2012).
}

El modelo clase invertida, al brindar mayor protagonismo al estudiantado y ofrecer espacios de interacción a través de estrategias educativas con apoyo de las TIC, atiende a la teoría constructivista de Vygotsky, en el cual las estrategias de aprendizaje se centran en él, y este aprendizaje se lleva a cabo por medio de una actividad cooperativa, a partir de la interacción con compañeros y docentes (Juca et al., 2017).

Herrera (2009), define al constructivismo como

una corriente que afirma que el conocimiento de todas las cosas es un proceso mental del individuo, resultado de un proceso de construcción o reconstrucción de la realidad que tiene su origen en la interacción entre las personas y el mundo (p. 1).

Por lo tanto, el aprendizaje se basa en la construcción de nuevos conocimientos a partir de conocimientos previos adquiridos en diversos entornos, que pueden ser sociales o educativos.

Considerando la teoría constructivista y las nuevas metodologías y estrategias didácticas, se sugiere un cambio en el papel de los agentes educativos, en el caso del estudiantado les ofrece el desarrollo de habilidades para estudios y entornos laborales futuros. Esto es posible si el estudiantado adquiere, a diferencia de la educación tradicional, un papel activo, autónomo, responsable de su aprendizaje y capaz de organizar y administrar sus tiempos para el logro de los objetivos planteados (López y Carmona, 2017).

$\mathrm{Al}$ implementar nuevas estrategias, en donde el estudiantado es el principal actor, es importante generar ambientes de confianza entre el estudiantado y el profesorado, brindando mejores canales de comunicación y gran convivencia, logrando de esta manera, interactuar y aprender en espacios colaborativos y enriquecedores, pues el estudiantado logra sentirse con gran apertura para participar constantemente.

De esta manera el profesorado juega un papel esencial, al convertirse en alguien que solo transmite conocimientos, sino que es guía, consejero e incluso, un cómplice que permite que el estudiantado descubra y aprenda, incluso que vaya más allá de los objetivos planteados (Tourón, Santiago, y Diez, 2014). También, se sugiere estimular en la participación del estudiantado a la hora de intervenir, promover la discusión guiada, ofrecer retroalimentación personalizada frecuentemente y atender dudas de manera oportuna (Fainholc, 2009).

El estudiantado pasa de ser un receptor pasivo de información a ser un constructor de conocimientos. Por lo tanto, el profesorado requiere de una mayor capacitación, tanto en conocimientos teóricos y procedimentales de la asignatura como de las competencias digitales necesarias para brindar el apoyo que, en 
su momento, pueda requerir el estudiantado (UNESCO, 2016). No obstante, el profesorado, es consciente de no ser la única fuente de información, el estudiantado busca por su propia cuenta y también aprende de otras fuentes, de esta manera, también se considera al profesorado como un aprendiz (Tourón et al., 2014).

\section{Procedimientos metodológicos}

De acuerdo con las necesidades y características del proyecto, se define el tipo de investigación que se llevó a cabo, considerando la metodología de la investigación - acción, la cual Rodríguez y Valldeoriola (2010), refieren como un proceso de transformar la realidad centrándose en un cambio educativo y un cambio social. El proceso se convierte en un ciclo en donde interviene una reflexión y una actividad transformadora.

La metodología de investigación - acción se trabajó en cuatro etapas, la primera se refiere al plan de acción que contempla el inicio del proyecto en el cual Elliot en Latorre (2005) describe como un aspecto problemático de la práctica profesional. Este plan de acción se constituye en tres aspectos: el problema o foco de investigación, el diagnóstico del problema o estado de la situación y la hipótesis o acción estratégica.

El foco de investigación se descubre a partir de la pregunta ¿Qué situación problemática de mi práctica profesional me gustaría mejorar?, identificando las áreas de oportunidad que se pueden investigar y cambiar desde el propio contexto. Para el diagnóstico se describe el problema de manera muy puntual, se obtienen evidencias para utilizarlas como punto de partida y como un elemento clave para la comparación con los resultados de la acción. También es importante la revisión documental, es decir, a partir de palabras clave y autores, recopilar investigaciones sobre el tema que se plantea investigar. Por último, la hipótesis, una vez desarrollada la investigación documental obtiene una idea de cómo se lleva a cabo la acción y los resultados que son posibles de obtener.

La segunda etapa es el desarrollo de la acción, en donde se lleva a cabo la puesta en marcha del cambio en la práctica profesional, en el caso del proyecto de clase invertida en la Preparatoria objeto de estudio, se efectuó la planeación de las actividades, así como el diseño, grabación y edición de los videos que forman parte de las secuencias didácticas propuestas. Una vez elaborado esto, continúa la implantación de los materiales en la plataforma educativa para su implementación con el estudiantado. Es importante considerar un cronograma de actividades que permitan establecer fechas de realización, por lo que la acción es controlada pero sujeta a cambios, en caso de no contar con los materiales necesarios, para llevarla a cabo, o la dinámica de grupo que se presente.

La tercera etapa es la observación de la acción, la que favorece ver lo que ocurre al poner en práctica el desarrollo de las actividades, lo cual en ocasiones requiere de cambios y ajustes dependiendo de los resultados que se presenten en el grupo. Los datos que se obtienen, brindan evidencias para su análisis y comprensión de si la mejora propuesta está teniendo lugar o no. Para el proyecto planteado, se utiliza la autoobservación de la acción. Se registran en un diario del profesor, las acciones llevadas a cabo, así como intenciones y motivos, así mismo se efectúan grabaciones de audio para tener evidencia de la acción dentro del aula de clase.

Por último, la cuarta etapa es la de la reflexión, la cual permite generar un círculo virtuoso orientado a procesos de diálogo, comprensión y mejora del proceso de enseñanza - aprendizaje, y con ello, trabajar desde comunidades de aprendizaje orientadas a interpretar la información, elaborar informes y plantear áreas de oportunidad para el inicio de un nuevo ciclo.

La investigación por desarrollar es de tipo intervención educativa, la cual se define como la acción intencional para la ejecución de acciones que conducen al logro del desarrollo integral del educando. El modelo de la intervención educativa es una metodología innovadora de investigación - acción. La importancia de este tipo de investigación es la contribución de los participantes, en este caso, fue por medio de un cuestionario diagnóstico el cual permitió conocer la percepción del estudiantado con respecto a las asignaturas de Informática. A partir de las respuestas analizadas, se llevará a cabo la intervención, con la finalidad de transformar y mejorar el proceso educativo. 
La intervención educativa se desarrolla con el estudiantado de primer semestre, de la Escuela Preparatoria Número Uno, en específico dentro de los grupos 1 ro. 7 y 1 ro. 9, considerando que aproximadamente se encuentran inscritos de entre 40 a 45 estudiantes por cada grupo, por lo tanto, un total aproximado de 90 estudiantes. El proceso de selección de la muestra fue intencional, pues fueron los grupos en los cuales la autora principal del trabajo impartía clases, en donde se ejecuta una evaluación diagnóstica para conocer las habilidades referentes a la competencia uso de la tecnología.

\section{ANÁLISIS Y DISCUSIÓN DE RESULTADOS}

En este apartado se observan las acciones que se llevaron a cabo para atender el problema por medio de la intervención educativa con un grupo de estudiantes de preparatoria que permitieron fortalecer la competencia el uso de la tecnología, la cual consistió en el diseño de actividades dentro de la asignatura de Informática 1, específicamente actividades de la unidad 3: Internet, considerando el modelo clase invertida.

La asignatura de Informática se cursa en los primeros cuatro semestres, y se imparte dos veces por semana, con una duración de dos horas cada sesión. En el caso específico de Informática 1, está conformada de cuatro unidades: Introducción, Sistemas Operativos, Internet y Software para mapas mentales y conceptuales.

Esta intervención se llevó a cabo dentro de las actividades de la unidad 3, debido a que los temas por revisar se enfocan en mayor medida a los requerimientos que marca la competencia uso de la tecnología. El estudiantado aprende a utilizar internet para la correcta búsqueda de información, para comunicación y colaboración dentro de los espacios virtuales. Sin embargo, no existe el tiempo suficiente para llevar a cabo prácticas que permitan profundizar en los correctos usos de este servicio.

En la unidad se trabajan conceptos básicos sobre el uso del internet, así como algunos de los servicios que ofrece, como correo electrónico, redes sociales, foros de discusión, blogs y la navegación en la biblioteca digital de la universidad. También se abordan los temas de búsquedas básicas y avanzadas, en donde se dan recomendaciones para una correcta búsqueda de información dentro de sitios confiables. Estos temas permiten fortalecer la competencia uso de la tecnología.

Para llevar a cabo la intervención educativa utilizando el modelo de clase invertida, apoyándose de las TIC, se consideraron los resultados del diagnóstico efectuado al estudiantado, con la finalidad de atender sus intereses y necesidades. A continuación, se describen dichas herramientas:

- Plataforma educativa Schoology (Friedman, Hwang, y Trinidad, 2018): un sistema de gestión de aprendizaje (LMS), es una herramienta que cuenta con varias opciones, en un sitio web, con fines educativos, por lo que se pueden crear cursos para cada grupo de estudiantes, en este caso se crea el curso Informática 1. Dentro del curso se concibieron carpetas, las cuales contienen las actividades de cada una de las unidades, también dentro de las carpetas se generaron cuestionarios que formaron parte de las actividades previas a la sesión de clase.

- Clips de Apple: es una aplicación para dispositivos con sistema operativo iOS, iPhone y iPad, que ofrece grabar videos con efectos muy vistosos, por lo que su utilidad es esencial para los videos de este proyecto, estos permiten presentar el contenido conceptual, a las y los estudiantes, de manera dinámica y divertida. Los videos se graban con iPhone $\mathrm{X}$, debido a que Clips, ofrece mayores efectos en este dispositivo (Apple, 2018).

- MovieMaker: el software de Microsoft para la edición de video con una interfaz intuitiva, lo que lo hace sencillo de utilizar, permite la inserción de imágenes, títulos, fragmentos de video, audio y créditos, además de la facilidad para definir los tiempos de duración de cada uno de los elementos a mostrar dentro del video (Microsoft, 2018). Se utiliza este software para la edición de los videos grabados en Clips. 
- YouTube: es una red social y un repositorio de videos para compartir y para que estén disponibles para otros usuarios. En este sitio, cada usuario puede crear su propio canal para publicar sus videos (YouTube, 2018). Para este proyecto, se crea el canal y se publican los videos grabados y editados con Clips y MovieMaker. Se copian los enlaces de los videos y se comparten dentro de las actividades de Schoology, con la finalidad de que el alumnado tenga el acceso a estos de manera rápida desde las actividades de la plataforma.

El uso de estas herramientas, para la creación de los videos y los espacios virtuales, no requirió de expertos en el área, pues cuentan con entornos de trabajos muy intuitivos y fáciles de utilizar, tanto para cada docente como para estudiante. Además, brinda resultados favorables con las opciones que ofrecen, considerando también que son herramientas de libre acceso.

- Secuencia didáctica

Para el diseño de la secuencia didáctica se utiliza un formato propio a partir de las propuestas presentadas por A. Díaz (2013b), Frade (2009) y Tobón, Pimienta y García (2010), los cuales mencionan que no se requiere de un seguimiento puntual de sus propuestas, sino más bien sugieren una identificación de las necesidades propias de cada docente, con respecto a las situaciones de aprendizaje que permitan el logro de los objetivos, anexando o quitando elementos dentro de su secuencia. Bajo este proceso, A. Díaz (2013a), define la secuencia didáctica como "un instrumento que demanda el conocimiento de la asignatura, la comprensión del programa de estudio y la experiencia y visión pedagógica del docente, así como sus posibilidades de concebir actividades para el aprendizaje de los alumnos" (p. 1).

En el caso propio del proyecto de intervención desarrollado, se plantea una secuencia didáctica bajo las necesidades de dicha intervención con el modelo clase invertida, en donde específicamente se incluye el apartado Preparación para la sesión, el cual brinda actividades previas a la sesión, en el que el estudiantado cuenta con videos con clases magistrales grabadas por el profesorado y pequeños cuestionarios para comprobar la revisión de los videos. Estas actividades permiten que el estudiantado llegue al salón de clases con conocimientos previos y teóricos, sobre el tema a abordar, dentro de la sesión presencial, y de esta manera aprovechar el tiempo en actividades prácticas y significativas.

En un primer momento, se diseñan tres secuencias didácticas para ser trabajadas en tres sesiones de dos horas cada una. En estas secuencias, se retoma la unidad 3 de la asignatura de Informática 1 , en donde se abordan temas sobre lo que es internet y los servicios que ofrece esta red. Para la intervención, se incorpora el diseño instruccional de estas secuencias a la plataforma Schoology, en donde se puede tener acceso a los videos.

- Evaluación

En la Preparación para la clase, se incluyen cuestionarios que fueron validados por expertos en el tema de las TIC y la didáctica en el nivel de Bachillerato, los cuales contesta el estudiantado en relación con los temas: 1) Uso del Internet, el cual está conformado por seis preguntas de opción múltiple, 2) Búsquedas en Internet, diseñado por cinco preguntas de opción múltiple, 3) Redes sociales, estructurado por cinco preguntas de opción múltiple, una vez que ha revisado el video con contenido teórico sobre el tema en cuestión, esto a través de una explicación en clase magistral. Estos cuestionarios permiten identificar los conocimientos, de los cuales se apropió cada estudiante, también, se logra un control de quienes sí han efectuado la observación del video, pues al responder los cuestionarios, el resultado de estos, queda registrado dentro de Schoology.

- Observación de la acción

La intervención educativa utilizando el modelo de clase invertida dentro de la Escuela Preparatoria Número Uno, se analiza bajo la estrategia de observación participante, la cual Latorre (2005), define como "la combinación de una serie de técnicas de obtención y análisis de datos entre las que se incluyen la observación y la participación directa" (p. 57). Se considera esta estrategia porque la 
investigadora resulta ser la docente de los grupos en los cuales se desea desarrollar la intervención e innovación educativa. En este proceso de observación, se tomaron en consideración las siguientes técnicas de recogida de información: diarios del profesor, cuestionarios y audios.

Los diarios del profesorado, son técnicas narrativas, que se han elaborado con la finalidad de recuperar información que permita complementar y fundamentar la investigación, por lo cual se recogen observaciones, reflexiones, interpretaciones, hipótesis y explicaciones de lo que ha ocurrido, en las clases, desde la perspectiva propia del profesorado, dado que tienen gran influencia sus estados de ánimo, sentimientos y creencias (Barba, González y Barba, 2014). Para esta investigación, es importante conocer el punto de vista del profesorado desde su práctica dentro del aula, lo que permite identificar las áreas de oportunidad o de mejora.

Para la identificación de los conocimientos adquiridos por parte del estudiantado, una vez revisados los videos con contenido teórico, se utilizan cuestionarios, en este caso, digitales elaborados por medio de la plataforma Schoology. Debido a que los videos se revisan como una actividad previa a la clase, se implementa el uso de estos cuestionarios para tener un control del conocimiento adquirido, por parte de los estudiantes, en cada uno de los temas vistos, así como la identificación del estudiantado que hace revisión del video.

Finalmente, para conocer las percepciones del estudiantado con respecto a la intervención efectuada, se procesan grabaciones de audio para obtener sus opiniones discutidas al finalizar las clases. Este tipo de técnica, Latorre (2005), las describe como las que "permiten captar la interacción verbal y registrar las emisiones con precisión” (p. 82).

- Reflexión

Para el término del primer ciclo del método Investigación - acción, se elabora una reflexión que tiene como objetivo presentar los resultados obtenidos en la intervención, estructurados en el uso de los videos, el modelo de clase invertida y la motivación; esto, con la finalidad de identificar áreas de oportunidad y hacer cambios para un futuro segundo ciclo de intervención.

Los videos grabados por la docente fueron subidos al canal en YouTube y compartidos dentro de la plataforma educativa Schoology, para que cada estudiante tuviera acceso a ellos. En la Figura 1, se representa la cantidad de estudiantes que están inscritos en los grupos en los que se aplicó la intervención, el total de ellos es de 72 , de los cuales, más de la mitad atendieron y efectuaron las instrucciones, previas a las sesiones, de manera puntual. Estas actividades consistían en revisar un video y contestar un cuestionario. Los resultados obtenidos fueron los que se presentan en el Gráfico $1:$ 


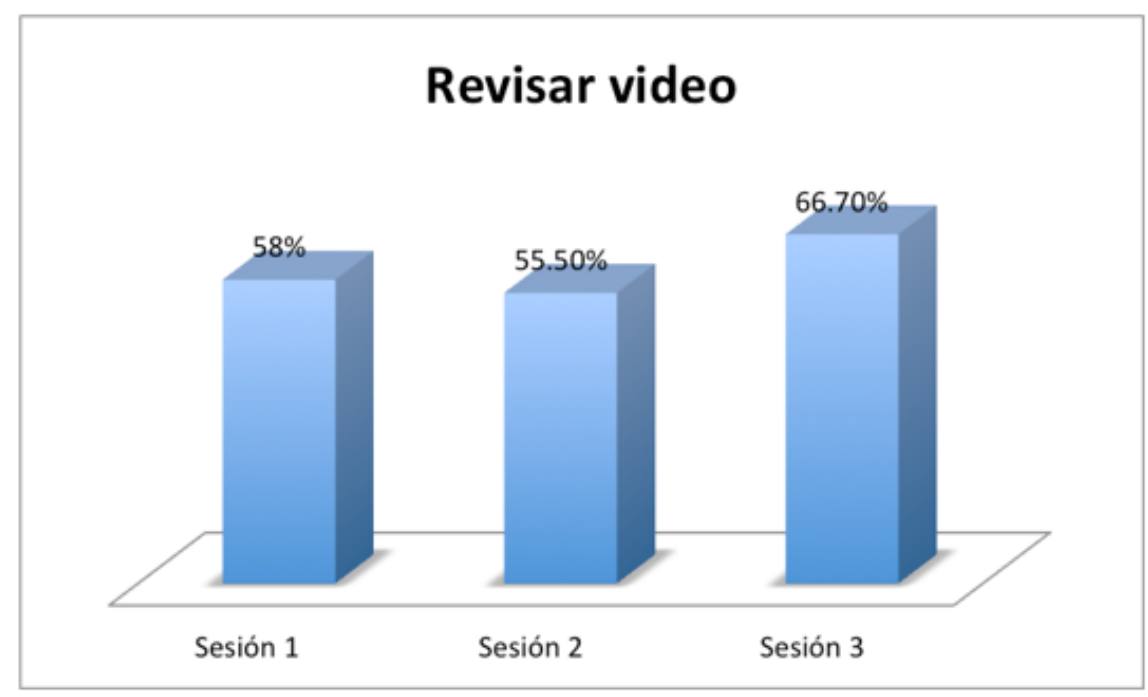

\section{GRÁFICO 1}

Fuente: Elaboración propia

Para la primera sesión, el 58\% del alumnado (42), en la segunda el 55.5\% (40) y en la tercera el 66.7\% (48). De la primera a la tercera sesión hubo un incremento del $8.7 \%$ en relación con el compromiso del alumnado, al inicio, solo lo atendieron 42 estudiantes y en la tercera sesión fueron 48, lo que confirma que el empleo del video como estrategia didáctica despertó el interés, además de que es un recurso creado por la docente del grupo, que se adapta a las necesidades y características del grupo, el cual pueden repetir cuantas veces requieran y les permite profundizar en la comprensión del tema.

En relación con la respuesta del cuestionario, en el Gráfico 2 se presentan los resultados obtenidos, que fueron los siguientes:

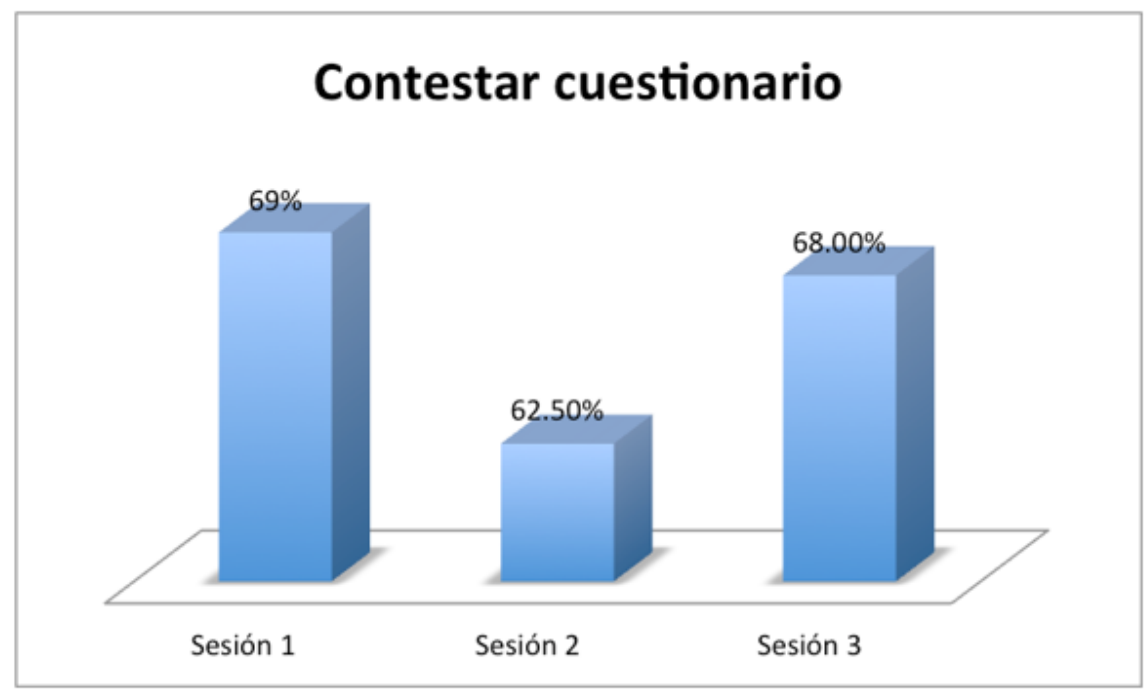

GRÁFICO 2

Fuente: Elaboración propia

Como se puede observar, el puntaje más alto lo obtiene la primera sesión, con un 69\%, le sigue la sesión 3 con un 68\%, y finalmente se encuentra la sesión 2 con un $62.05 \%$. Es interesante reflexionar en torno a los resultados, pues la primera sesión resulta ser la que obtiene mayor participación en esta actividad, lo cual 
refleja el interés del estudiantado por continuar con el proceso de articulación entre ver el video y contestar el cuestionario correspondiente, existe en la segunda sesión una baja considerable que corresponde a 6.5\%, la cual se incrementa nuevamente en la tercera sesión con $5 \%$, pero que no alcanza el nivel de respuesta de la primera sesión, entonces, ¿qué tipo de actividades, seguimiento y proceso de mediación se requiere trabajar para que el estudiantado incremente su participación?, otra pregunta también relevante es ¿qué pasa en la tercera sesión que el nivel de participación se incrementa?, ¿cómo lograr que el porcentaje de participación vaya en aumento sesión tras sesión?

Con respecto a la percepción del estudiantado sobre los videos, se llevó a cabo un análisis cualitativo, por lo que se realizaron preguntas abiertas y generales como: ¿Qué les pareció esta nueva forma de trabajar? en dónde visualizan videos previos a la clase, estas y las respuestas fueron grabadas en audio para su recopilación. En la Figura 2, se observan las opiniones del alumnado, en donde indican que tener los videos les permite repetir la información las veces que ellos lo consideren necesario, además de encontrarlos divertidos. En cuanto a contenido, encontraron la información clara y concisa, dado que se buscó presentarla en tiempos cortos, de 3 a 5 minutos.

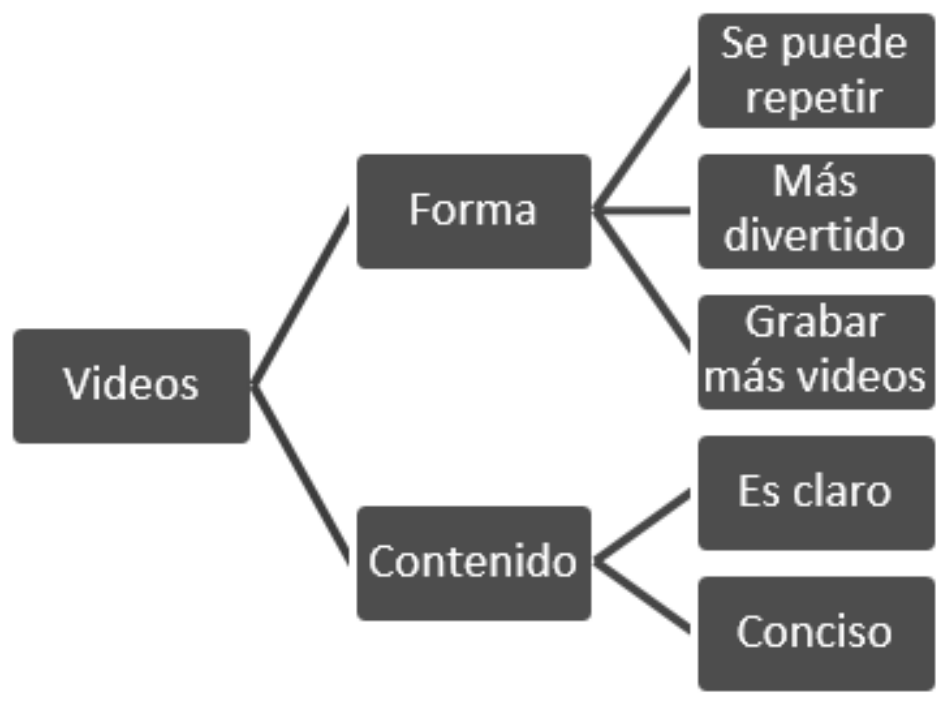

FIGURA 2

Percepción de las y los estudiantes sobre los videos. Fuente: Elaboración propia

El estudiantado se mostró motivado con la presentación de los videos, incluso propuso grabar sus propios videos para actividades posteriores, apoyándose del software utilizado por su docente. Para la recopilación de más opiniones, se desarrolló un cuestionario en línea con las preguntas del diagnóstico inicial y se anexó dos más para indagar en la percepción, del alumnado, sobre el uso de los videos y la clase invertida. Los resultados de las preguntas se presentan en la Tabla 1. 
TABLA 1

Respuestas de las y los estudiantes sobre su percepción y conocimientos adquiridos en la intervención con clase invertida

\begin{tabular}{|c|c|}
\hline Categorización & Respuestas de las y los estudiantes \\
\hline \multirow[t]{2}{*}{ Estrategias } & $\begin{array}{l}\text { Gustaron: videos, prácticas, } \\
\text { plataforma educativa schoology, } \\
\text { lluvias de ideas. }\end{array}$ \\
\hline & $\begin{array}{l}\text { No gustaron: realizar apuntes, tareas } \\
\text { y actividades largas. }\end{array}$ \\
\hline Habilidades y conocimientos & $\begin{array}{l}\text { Mapas mentales y conceptuales } \\
\text { Internet y búsquedas de información }\end{array}$ \\
\hline Integración de TIC & $\begin{array}{l}\text { Clases más dinámicas Reducción de } \\
\text { papel Facilita el trabajo }\end{array}$ \\
\hline TIC para búsqueda de información & $\begin{array}{l}\text { Google, navegador, celular, } \\
\text { computadora, caracteres especiales }\end{array}$ \\
\hline $\begin{array}{l}\text { TIC para administración de } \\
\text { información }\end{array}$ & $\begin{array}{l}\text { Mapas mentales y conceptuales } \\
\text { Carpetas }\end{array}$ \\
\hline Aplicaciones utilizadas & $\begin{array}{l}\text { Ubuntu, Xmind, Canva, Recortes, } \\
\text { Schoology, CmapTools, Navegadores }\end{array}$ \\
\hline Sugerencias & $\begin{array}{l}\text { Más juegos, prácticas, más tiempo } \\
\text { en actividades complejas }\end{array}$ \\
\hline \multirow[t]{2}{*}{ Clase invertida } & $\begin{array}{l}\text { Videos: buenos, se comprendieron } \\
\text { mejor los temas, aprender desde } \\
\text { casa }\end{array}$ \\
\hline & $\begin{array}{l}92 \% \text { prefiere la clase invertida } 8 \% \\
\text { prefiere metodología tradicional }\end{array}$ \\
\hline
\end{tabular}

Fuente: Elaboración propia

Estos resultados muestran las habilidades que el estudiantado ha adquirido, en cuanto a conocimientos teóricos sobre la competencia uso de la tecnología, al mencionar software para elaborar mapas conceptuales y mentales, así como el software necesario para hacer búsquedas básicas y específicas en Internet. Hacen la sugerencia de agregar más dinámicas y juegos a las clases, por lo que se consideran en intervenciones posteriores.

En el caso de los videos, como se menciona en párrafos anteriores, el estudiantado los ha aceptado de manera favorable haciendo comentarios sobre el poder aprender desde casa, una mejor comprensión de los temas y presentación de estos de forma divertida. Con respecto a la preferencia de la metodología tradicional con respecto a la clase invertida, un $92 \%$ prefiere la clase invertida y un $8 \%$ se queda con la tradicional.

Estos resultados permiten mostrar los beneficios del uso del modelo de clase invertida, sin embargo, existen elementos que es importante continuar trabajando, como es el caso de mayor calidad y organización en los videos, la diversificación de aplicaciones para el diseño y desarrollo de los videos, la identificación de otras estrategias lúdicas para las actividades dentro del aula, así como la creación de más instrumentos de evaluación que permitan observar la apropiación de la competencia uso de la tecnología.

\section{Conclusiones}

El método de clase invertida resultó ser una transformación con resultados favorables para atender y combatir a las problemáticas identificadas en el entorno educativo, dentro de la asignatura de informática 1, en la Escuela Preparatoria Número Uno. Se logró atraer la atención de las y los estudiantes con la grabación de videos por la docente con contenido teórico y práctico, para una mejor comprensión de los temas. De esta manera se alcanza el objetivo general del proyecto, una propuesta de intervención educativa para el desarrollo de la competencia uso de la tecnología. 
Se identificaron las dimensiones de la competencia uso de la tecnología, del modelo curricular de la UAEH, para la elaboración de las planeaciones didácticas, estas consideraron también las estrategias didácticas propuestas en el modelo como el trabajo colaborativo, el uso de TIC y la diversidad de escenarios, tanto virtuales como de aula y reales, en donde se plantean problemáticas del contexto en el cual cada estudiante se encuentra.

La investigación sobre intervenciones mediadas con la clase invertida, así como referentes teóricos sobre este modelo, permitió conocer resultados, áreas de oportunidad, ventajas y requerimientos para el diseño, desarrollo e implementación de este proyecto. Sin duda, tomar en cuenta trabajos previos de otras instituciones, incluso de otros países enriqueció y permitió mejorar la intervención, en aspectos como la duración de los videos, las aplicaciones utilizadas, los medios de presentación de los materiales y las estrategias didácticas llevadas a cabo.

Una de las principales problemáticas identificadas en el estudiantado, de primer semestre de la institución, es el índice de reprobación en la asignatura Informática 1. Tomando referentes de la Dirección General de Planeación de la UAEH (2017), se encontró que el porcentaje incrementó un 19.61\% del 2008 al 2017, en el periodo Julio Diciembre. Debido a esto, surge la necesidad de brindar a las y los estudiantes espacios de aprendizaje más dinámicos, que permitan una mayor motivación y de esta manera lograr el desarrollo de competencias y, a su vez, la permanencia en sus estudios de Nivel Medio Superior.

Esta problemática buscó ser combatida con el uso del modelo de clase invertida y las TIC para actividades correspondientes a la unidad 3 de la asignatura Informática 1, por lo que se diseñaron tres secuencias didácticas que incluyen el apartado Preparación para la sesión, el cual consiste en la revisión de un video con contenido teórico y/o práctico del tema en cuestión, así como un pequeño cuestionario para llevar un control de los que desarrollan la actividad y la comprensión del contenido.

Posterior a eso, en clase se atendieron dudas sobre el contenido de los videos y se llevaron a cabo diversas actividades como prácticas con la computadora, discusiones guiadas y sociodramas, para una mejor comprensión de los temas. Los resultados sobre la percepción de esta nueva forma de aprender, por parte de las y los estudiantes, fue positiva, ya que sus comentarios y disposición dentro del aula permitieron observar motivación en ellos.

La ejecución de las actividades previas a la sesión en aula, presentan un $66.7 \%$ de participación, lo que deja ver que más de la mitad revisaron el video y contestaron el cuestionario, por lo que llegaban al salón con más conocimientos previos sobre el tema a presentar y generando mayor participación en las discusiones. En una encuesta final, se les preguntó sobre la preferencia del método tradicional y la clase invertida, el $92 \%$ de las y los estudiantes considera más interesante la clase invertida.

\section{ReFERENCiAS}

Apple. (2018). Clips. Recuperado de Apple: https://www.apple.com/mx/clips/

Barba, J. J., González, G. y Barba, R. A. (2014). El uso de los diarios del profesorado como instrumento de reflexiónsobre-la-acción. Revista Española de educación física y deportes (405), 55-63.

Barros, V. M. y Martínez, M. B. (2018). Aula invertida en la enseñanza de Álgebra en la educación superior. Espirales, 2(13), 1-14.

Bergmann, J. y Sams, A. (2012). Flip your classroom. Colorado, United States of America: ISTE ASCD.

Cabero, J. y Ruiz, J. (2017). Las Tecnologías de la Información y la Comunicación para la inclusión. International Journal of Educational Research and Innovation, (9), 16 - 30. Recuperado de https://bit.ly/2mJavNq

Churches, A. (2009). Taxonomía de Bloom para la era digital. Eduteka. Recuperado de http://eduteka.icesi.edu.co/ articulos/TaxonomiaBloomDigital

Díaz, Á. (2013a). Guía para la elaboración de una secuencia didáctica. Comunidad de conocimiento. UNAM, 1-15.

Díaz, Á. (2013b). TIC en el trabajo del aula. Impacto en la planeación didáctica. RIES - UNIVERSIA, 4(10), 3-21. 
Díaz F. (2010). Los profesores ante las innovaciones curriculares. Revista Iberoamericana de Educación Superior, 1(1), $37-57$.

Fainholc, B. (2009). Redefinición del rol del profesor en propuestas de aprendizaje mixto. Revista mexicana de bachillerato a distancia, 2(3), 74-85.

Ferriz, A., Sebastiá, S. y García, S. (2017). Clase invertida como elemento innovador en educación física: efectos sobre la motivación y la adquisición de aprendizajes en primaria y bachillerato. En R. Roig Vila, Investigación en docencia universitaria. DIseñando el futuro a partir de la innovación educativa (pp. 211-222). España: Octaedro.

Frade, L. (2009). Desarrollo de competencias en educación: desde preescolar hasta el bachillerato. México: Inteligencia educativa.

Friedman, J., Hwang, R. y Trinidad, T. (2018). Schoology. Recuperado de https://www.schoology.com/

García, A. (2013). El aula inversa: cambiando la respuesta a las necesidades de los estudiantes. Avances en supervisión educativa, (19), 1-8. Recuperado de https://avances.adide.org/index.php/ase/article/view/118

Gómez, J. E. (2016). Las TIC como puente cognitivo en el triángulo didáctico. Miradas, 1(14), 122-131. doi: https: //dx.doi.org/10.22517/25393812.15581

González, M., Martínez, K., González, A., y Bernal, J. (2015). Importance of instructional design for the implementation of Flipped Learning Model in the modality of distance education. Ecorfan, 2(3), 140-160.

Herrera, Á. M. (2009). El constructivismo en el aula. Innovación y experiencias educativas, (14),1-10.

Ibáñez, C. (2007). Un análisis crítico del modelo del triángulo pedagógico. Una propuesta alternativa. Revista Mexicana de Investigación Educativa, 12(32), 435-456.

Jordán, C., Pérez, M. J. y Sanabria, E. (2014). Investigación del impacto en el aula de matemáticas al utilizar flip education. Experiencias Docentes, 4(2), 9-22.

Juca, F. J., Burgo, O. B., Fadúl, J. S., García, M. B., Cruz, J. C. y Vélez, L. (2017). La educación invertida. Un nuevo reto para la educación superior. Estudios Pedagógicos Originales, 40-45.

Latorre, A. (2005). La investigación-acción Conocer y cambiar la práctica educativa. España: Graó.

López, H. y Carmona, H. (2017). El uso de las TIC y sus implicaciones en el rendimiento de los alumnos de bachillerato. Un primer acercamiento. Education in the Knowledge Society, 18(1), 21-38. doi:https://dx.doi.or $\mathrm{g} / 10.14201 / \mathrm{eks} 20171812138$

Madrid, E. M., Angulo, J., Prieto, M. E., Fernández, M. T. y Olivares, K. M. (2018). Implementación del aula invertida en un curso propedéutico de habilidad matemática en bachillerato. Apertura, 10(1), 24-39. doi: https://dx.doi .org/10.18381/Ap.v10n1.1149

Martín, D. y Santiago, R. (2016). Flipped Learning en la formación del profesorado de secundaria y bachillerato. Formación para el cambio. Contextos educativos Revista de Educación, 0, 117-134. doi: https://doi.org/10.181 $72 /$ con. 2854

Massut, M. F. (2015). Estudio de la utilización de vídeos tutoriales como recurso para las clases de matemáticas en el bachillerato con "Flipped Classroom". Barcelona, España: Universidad de Barcelona.

Melo, L. y Sánchez, R. (2017). Análisis de la percepción de los alumnos sobre la metodología Flipped Classroom para la enseñanza de técnicas avanzadas en laboratorios de análisis de residuos de medicamentos veterinarios y contaminantes. Educación Quimica, 28(1), 30-37. doi: https://dx.doi.org/10.1016/j.eq.2016.09.010

Microsoft. (2018). Movie Maker 10 - Tell Your Story. Recuperado de https://bit.ly/2NUR1yS

Montenegro, J. C. y González, G. E. (2017). Aula invertida en el proceso académico en la asignatura de historia para los alumnos del segundo año de bachillerato de la unidad educativa patria ecuatoriana diseño de un sitio web. Guayaquil, Ecuador: Universidad de Guayaquil.

Rodríguez, D. y Valldeoriola, J. (2010). Metodología de la Investigación. Cataluña, España: Universitat Oberta de Catalunya.

Salas, F. E. (2016). Aportes del modelo de Yrjö Engeström al desarrollo teórico de la docencia universitaria. Revista Educación, 40(2) 1-22. doi: https://dx.doi.org/10.15517/revedu.v40i2.15257

Sánchez, R. (2017). Aula invertida, metodología del siglo XXI. Islas Baleares, España: Universidad de las Islas Baleares. 
Tobón, S., Pimienta, J. H. y García, J. A. (2010). Secuencias didácticas: aprendizaje y evaluación de competencias. México: Pearson.

Tourón, J., Santiago, R. y Diez, A. (2014). The Flipped Classroom: Cómo convertir la escuela en un espacio de aprendizaje. Grupo Océano.

UNESCO. (2016). Competencias y estándares TIC desde la dimensión pedagógica: Una perspectiva desde los niveles de apropiación de las TIC en la práctica educativa docente. Cali, Colombia: Pontificia Universidad Javeriana.

Valero, S. (2017). Poniendo del revés la clase de TIC de 1ro. de bachillerato. Castellón, España: Universidad Jaime I.

Vaughan, N., Cleveland, M. y Garrison, D. (2013). Teaching in Blended Learning Environments. Au Press.

YouTube. (2018). YouTube. Recuperado de https://www.youtube.com/?hl=es-419\&gl=MX

\section{BY-NC-ND}

